\title{
Analysis of risk factors, maternal and fetal outcome of spontaneous preterm premature rupture of membranes: a cross sectional study
}

\author{
Surekha S. Mohan*, Chamaraja Thippeveeranna, Naorem N. Singh, Laiphrakpam R. Singh
}

Department of Obstetrics and Gynecology, Regional Institute of Medical Sciences, Imphal, Manipur, India

Received: 05 July 2017

Accepted: 22 July 2017

*Correspondence:

Dr. Surekha S. Mohan,

E-mail: chami19854@gmail.com

Copyright: () the author(s), publisher and licensee Medip Academy. This is an open-access article distributed under the terms of the Creative Commons Attribution Non-Commercial License, which permits unrestricted non-commercial use, distribution, and reproduction in any medium, provided the original work is properly cited.

\begin{abstract}
Background: Preterm premature rupture of membranes (PPROM) is one of the most common complications of pregnancy. It is an important cause of perinatal morbidity and mortality. Objective of present study was to determine incidence, risk factors, maternal, fetal outcome of PPROM occurring in patients attending a tertiary hospital in North Eastern India.

Methods: This descriptive cross- sectional study was carried out in the department of Obstetrics and Gynecology, Regional Institute of Medical Sciences, Imphal, India. Out of 15,969 deliveries between July 2010 to December 2011, 358 pregnant patients with spontaneous preterm premature rupture of membranes from 28-0 to 36-6weeks gestation were reviewed. After excluding patients with uterine anomalies, intrauterine deaths and congenital anomalies 293 patients were included and evaluated in this study.

Results: The incidence of PPROM was $2.2 \%$. Out of 293 patients $86 \%$ were singleton pregnancies, $12.9 \%$ were twins and $1.02 \%$ were triplets. $48.4 \%$ had previous history of termination of pregnancy, $28.6 \%$ history of previous PPROM and $16.3 \%$ had urinary tract infection. The mean gestational age at the onset of membrane rupture was $34.1+2.4$ weeks and the latency from the membrane rupture to delivery interval ranged from 0-72 days with a mean of 48.4 hours. There were 7 stillbirths $(2.38 \%)$ and 4 neonatal deaths $(1.02 \%)$ resulting in perinatal deaths of $3.29 \%$ and perinatal mortality rate of 0.329 per 1000 births due to PPROM. Maternal morbidity was minimal with postpartum haemorrhage in 11 patients $(4.1 \%)$, abruptio placentae in 7 patients $(2.3 \%)$ and sepsis in 43patients (14.6\%). 66 patients $(22.5 \%)$ underwent caesarean section for which malpresentations were the major cause.

Conclusions: Despite remarkable advances in perinatal care, preterm premature rupture of membranes continues to cause perinatal morbidity and mortality. Strategies should be developed for its prevention. Management protocol should be improved in regard to vaginal swab culture and use of specific antibiotics.
\end{abstract}

Keywords: Preterm premature rupture of membranes, Perinatal morbidity, Mortality

\section{INTRODUCTION}

Premature rupture of membranes (PROM) is defined as spontaneous membrane rupture that occurs before the onset of labour. When spontaneous membrane rupture occurs before 37 weeks gestation, it is referred to as preterm premature rupture of membranes (PPROM). ${ }^{1}$ PPROM complicates approximately $2 \%-3 \%$ of all pregnancies below 37 weeks gestation. ${ }^{2}$ P PROM complicates one-third of preterm births and is responsible for significant perinatal morbidity and mortality, largely related to prematurity. ${ }^{3}$

There are numerous risk factors for PPROM, such as physiologic changes, intrauterine infection at early gestational age, lower socioeconomic status of pregnant women, inadequate prenatal care and inadequate nutrition during pregnancy, sexually transmitted infections, 
vaginal bleeding and smoking during pregnancy etc. ${ }^{4}$ Subclinical intrauterine infection has been implicated as a major aetiological factor in the pathogenesis and consequential maternal and neonatal morbidity in PPROM. ${ }^{5}$ Education plays a significant role in reducing the risk of PROM especially in developing countries. ${ }^{2}$

Preterm PROM is an important cause of perinatal morbidity and mortality, particularly because it is associated with brief latency from membrane rupture to delivery, perinatal infection, and umbilical cord compression due to oligohydramnios.

The risk of these complications increases with decreasing gestational age at membrane rupture. Respiratory distress syndrome (RDS) is the most common serious complication after preterm PROM at any gestation. Other serious acute morbidities including necrotizing enterocolitis, intraventricular haemorrhage, and sepsis are common with early preterm birth but relatively uncommon near term. Remote from term, serious perinatal morbidity that may lead to long-term sequelae or death is common. ${ }^{6}$ Probable maternal complications are chorioamnionitis (3-30\%), endometritis, abruption placenta. Recurrence of PROM may occur in $20 \%$ cases. $^{2}$

PPROM is very common in the obstetric wards. We face problem in diagnosis, monitoring and adopting treatment policy. The aim of this study is to determine incidence, risk factors and to see the maternal and fetal outcome of preterm premature rupture of the membranes. It will give an opportunity to analyse the magnitude of problems caused by PPROM.

\section{METHODS}

This descriptive cross- sectional study was carried out in the department of Obstetrics and Gynaecology, Regional Institute of Medical Sciences, Imphal, Manipur, India between July 2010 to December 2011. Out of 15,969 deliveries between July 2010 to December 2011, 358 pregnant patients with spontaneous preterm premature rupture of membranes from 28-0 to 36-6weeks gestation were reviewed.

After excluding women with pregnancy 37 completed weeks, with established labour, with ante partum haemorrhage, uterine anomalies, intrauterine death and foetuses with congenital anomalies, two hundred and ninety-three in patients with preterm premature rupture of membranes were recruited in this study. Both primi and multi gravid women, who consented to participate in this study, whose pregnancy duration 28-0 weeks to 36-6 weeks, with spontaneous rupture of the membrane, not in active labour were included in this study.

Detailed history regarding the age, parity, socioeconomic status, duration of pregnancy, time and onset of rupture of membranes, past history of rupture of membranes, past obstetric history was taken. Rupture of the membrane was diagnosed by history of a gush of fluid from the vagina or continued leakage of fluid from the vagina. A full clinical examination regarding pulse, BP, temperature, abdominal palpation to confirm presentation, uterine contraction and fetal heart sound.

Demonstration of membranes rupture was made by a sterile speculum examination visualizing flow of amniotic fluid from the cervical os and/or it's pooling in posterior vaginal fornix spontaneously or by fundal pressure and demonstrating alkaline $\mathrm{PH}$ of vaginal fluid by litmus paper. Cervical dilatation and effacement was assessed at the same time. Gestational age was determined from LMP and from early USG scan.

Investigations like haemoglobin, total leucocyte count, differential leucocyte count, urine routine was done in all cases. After the maternal condition was stabilised USG was done in selected cases. Plan of management was decided on gestational age, cervical condition, latent period, presentation of the fetus, symptoms and signs of infection.

All patients were given antibiotics. Inj ceftriaxone + sulbactum was given IV 12th hourly for 3 days later it was switched over to oral antibiotics which was continued for 7 days. Two doses of betamethasone $12 \mathrm{mg}$ IM was given $24 \mathrm{hrs}$ apart for patients with gestation less 34 weeks. Few patients who showed uterine contraction short term tocolysis was given in order to allowed steroid therapy which can produce maximal effect on pulmonary maturation.

Fetal surveillance was checked by daily fetal kick count and auscultation of fetal heart sound 6th hourly. Maternal monitoring to detect the sign of chorioamnionitis was done by recording of pulse, blood pressure, temperature, fundal height, abdominal tenderness, colour and smell of liquor and fetal conditions sixth hourly. Fetal growth liquor volume was assessed every 3 to 4 weeks by ultrasound.

Since our hospital was a tertiary centre and many cases were referred cases from primary health centres, community health centres, sub centres etc where antibiotics were started randomly to all patients. Performing swab culture was not consistent with the management protocol of Department of Obstetrics and Gynaecology in our hospital

Patients with features of chorioamnionitis which included maternal temperature above $1000 \mathrm{~F}$, maternal tachycardia, fetal tachycardia (fetal heart rate>160 beat/minute), uterine tenderness, foul smelled vaginal discharge and maternal leucocytosis $(>16000 / \mu \mathrm{L})$ was taken as the indication of termination of pregnancy.

Patients with features of chorioamnionitis were given broad spectrum antibiotics in parental route during labour. Antibiotic was given to the baby after delivery in 
such cases. All the neonates were referred to neonatal ward for further management according to the hospital protocol.

Without chorioamnionitis, a conservative approach was taken, advice for bed rest, to wear a sterile pad which was inspected every sixth hourly to detect any change of colour of liquor and also to document amount of loss.

If patient developed signs and symptoms of infection or conservative approach failed then pregnancy was terminated by induction, augmentation or caesarean section.

The labour was induced with dinoprostone gel or augmented with oxytocin drip if there was no contraindication or underwent caesarean section.

\section{Statistical analysis}

Data were collected by standard questionnaire from the allocated patients. All data was checked and edited after collection and analysed statistically by computing proportions and percentages. The statistical inference was obtained by computing chi square test for difference between any two values and considered statistically significant if the $\mathrm{P}$ value was $<0.005$.

\section{RESULTS}

The incidence of PPROM was $2.2 \%$. The maternal and fetal outcome of PPROM was analysed in relation to age, parity, socioeconomic status, antenatal care, gestational age, past obstetric history, mode of delivery.

Table 1 shows various demographic variables and its relation in patients with PPROM. Highest number of PPROM cases $(50.1 \%)$ occurred in the age group between 20-30years.

Sixty one percent of the patients were multiparous where as $38.9 \%$ were primigravida. Seventy two percent of patients were booked cases (at least three antenatal check-ups with at least one in the third trimester).

Fifty five percent of patients had an income of $<4000$ per month. Twenty one percent were uneducated and $40.2 \%$ were educated up to secondary level.

Highest number of cases (56.9\%) with PPROM occurred near to term (34-0 to 36-6weeks). The mean gestational age at the onset of membrane rupture was 34.1 weeks.

$12.9 \%$ were twins and $1.02 \%$ were triplets. $48.4 \%$ had previous history of termination of pregnancy, $28.6 \%$ history of previous PPROM, 32.7\% had lower genital tract infection and $16.3 \%$ had urinary tract infection. Cervical effacement at the time of admission was $0-50 \%$ in $66.6 \%$ of the patients and dilatation $<2 \mathrm{~cm}$ in $72 \%$ of the patients.
Table 1a: Incidence of PPROM in hospital delivered group in this study and Maternal demographic variables.

\begin{tabular}{|c|c|c|}
\hline Total no. of deliveries & $\begin{array}{l}\text { No. of PPROM } \\
\text { cases }\end{array}$ & $\%$ \\
\hline 15,969 & 358 & 2.2 \\
\hline \multicolumn{3}{|l|}{ Maternal demographic factors } \\
\hline \multicolumn{3}{|l|}{ Age in years } \\
\hline$<20 \mathrm{yrs}$ & 53 & 18 \\
\hline 20-30yrs & 147 & 50.1 \\
\hline$>30 y r s$ & 93 & 31.7 \\
\hline \multicolumn{3}{|l|}{ Parity } \\
\hline Prime & 114 & 38.9 \\
\hline 1 & 83 & 28.3 \\
\hline 2 & 41 & $14 \%$ \\
\hline 3 & 36 & 12.3 \\
\hline$\geq 4$ & 19 & 6.5 \\
\hline \multicolumn{3}{|l|}{ Antenatal care } \\
\hline Booked & 211 & 72 \\
\hline Unbooked & 82 & 27.9 \\
\hline \multicolumn{3}{|l|}{ Socioeconomic status } \\
\hline Low & 162 & 55.2 \\
\hline Middle & 87 & 29.6 \\
\hline High & 46 & 15.6 \\
\hline \multicolumn{3}{|l|}{ Education } \\
\hline Uneducated & 64 & 21.8 \\
\hline Primary & 92 & 31.3 \\
\hline Secondary & 118 & 40.2 \\
\hline Graduate & 19 & 6.4 \\
\hline \multicolumn{3}{|l|}{ Gestational age } \\
\hline $28-0$ to $31-6$ weeks & 32 & 10.9 \\
\hline $32-0$ to $33-6$ weeks & 96 & 32.7 \\
\hline $34-0$ to $36-6$ weeks & 167 & 56.9 \\
\hline \multicolumn{3}{|l|}{ No. of foetuses } \\
\hline Single & 252 & 86 \\
\hline Twins & 38 & 12.9 \\
\hline Triplets & 3 & 1.02 \\
\hline \multicolumn{3}{|l|}{ Past obstetric history } \\
\hline History of spontaneous abortic & 52 & 17.7 \\
\hline History of PPROM & 84 & 28.6 \\
\hline $\begin{array}{l}\text { History of preterm delivery } \\
\text { due to PROM }\end{array}$ & 36 & 12.2 \\
\hline $\begin{array}{l}\text { History of termination of } \\
\text { pregnancy }\end{array}$ & 142 & 48.4 \\
\hline \multicolumn{3}{|l|}{ Cervical condition } \\
\hline Cervical effacement $0-50 \%$ & 195 & 66.6 \\
\hline $51-100 \%$ & 98 & 33.4 \\
\hline Cervical dilatation $<2 \mathrm{~cm}$ & 211 & 72.01 \\
\hline$>2 \mathrm{~cm}$ & 82 & 27.98 \\
\hline \multicolumn{3}{|l|}{ Associated conditions } \\
\hline Urinary tract infection & 48 & 16.3 \\
\hline Anaemia & 38 & 12.9 \\
\hline Lower genital tract infection & 52 & 29.05 \\
\hline Hypertension & 18 & 6.1 \\
\hline Diabetes mellitus & 2 & 1.03 \\
\hline Renal disease & 1 & 0.3 \\
\hline
\end{tabular}


Table 1b: Incidence of PPROM in hospital delivered group in this study and Maternal demographic variables.

\begin{tabular}{|lll|}
\hline Mode of delivery & $\begin{array}{l}\text { No. of PPROM } \\
\text { cases }\end{array}$ & $\%$ \\
\hline $\begin{array}{l}\text { Normal vaginal delivery } \\
\text { Assisted vaginal delivery } \\
\text { (forceps/ventouse) }\end{array}$ & 189 & 64.5 \\
\hline $\begin{array}{l}\text { Breech delivery } \\
\text { (spontaneous/assisted) }\end{array}$ & 8 & 2.7 \\
\hline LSCS & 66 & 10.2 \\
\hline PPROM to delivery interval in hours & 22.5 \\
\hline <24 & 56 & 19.11 \\
\hline $24-48$ & 104 & 35.4 \\
\hline $49-72$ & 57 & 19.4 \\
\hline$>72$ & 76 & 25.9 \\
\hline Indications for LSCS & & \\
\hline Breech & 26 & 39.3 \\
\hline Transverse lie & 5 & 7.5 \\
\hline Fetal distress & 11 & 16.6 \\
\hline Oligohydramnios & 9 & 13.6 \\
\hline Previous LSCS & 7 & 10.6 \\
\hline Failure of induction & 5 & 7.5 \\
\hline Non-progress of labour & 3 & 4.5 \\
\hline
\end{tabular}

In this study $77.5 \%$ had vaginal delivery, among which $64.5 \%$ had normal vaginal delivery.

$22.5 \%$ underwent LSCS. In this study $54.5 \%$ delivered within 48 hours, $19.4 \%$ between $49-72$ hours and $25.9 \%$ after 72 hours. The main indications for LSCS in this study were malpresentations and fetal distress.

The $\mathrm{P}$ value of $<0.001$ which was statistically significant for the mode of delivery according to Bishop's score.

Maximum number of LSCS (39.4\%) were seen in the group with bishop's score $0-5$.

There was no statistical significance among primigravida and multigravida. Total number of LSCS was 66 among which maximum number $(62.1 \%)$ was among multigravida.

Maternal morbidity was found in 51 cases $(17.4 \%)$ with postpartum haemorrhage in eleven patients $(4.1 \%)$, abruptio placentae in seven patients $(2.3 \%)$ and sepsis in forty-three patients (14.6\%). The maternal morbidity increased as the duration of PPROM increases. There was statistical significance found in this analysis.

Table 2: Analysis of PPROM according to Bishop's score at the time of admission and mode of delivery.

\begin{tabular}{|lllllll|}
\hline Bishops score & No. of cases & Vaginal delivery & $\%$ & LSCS & \% & P value \\
\hline $0-5$ & 142 & 84 & 59.1 & 56 & 39.4 & 0.0015 \\
\hline $6-10$ & 123 & 113 & 91.8 & 10 & 8.1 & - \\
\hline $11-13$ & 28 & 28 & 100 & - & - & \\
\hline
\end{tabular}

Table 3: Analysis of PPROM according to mode of delivery and parity.

\begin{tabular}{|llllll|l|}
\hline Mode of delivery & No. of cases & Primgravida & $\%$ & Multigravida & $\%$ & P value \\
\hline Normal vaginal delivery & 189 & 78 & 41.2 & 111 & 58.7 \\
\hline $\begin{array}{l}\text { Assisted vaginal } \\
\text { delivery(forceps/ventouse) }\end{array}$ & 8 & 5 & 62.5 & 3 & 37 & 0.07 \\
\hline Breech delivery (spontaneous/assisted) & 30 & 6 & 20 & 24 & 80 \\
\hline LSCS & 66 & 25 & 37.8 & 41 & 62.1 \\
\hline
\end{tabular}

Out of 334 babies delivered perinatal morbidity was seen in 75 cases and mortality in 11 cases.

The major morbidity was due to sepsis followed by birth asphyxia and RDS. The main cause of perinatal mortality in this study was sepsis $1.49 \%$ followed by birth asphyxia and RDS.

In this study, perinatal mortality was highest $25 \%$ when birth weight was upto $1000 \mathrm{gms}$. As the birth weight increases perinatal morbidity and mortality decreases.
Table 4: Analysis of PPROM according to duration of PPROM and maternal morbidity.

\begin{tabular}{|c|c|c|c|}
\hline $\begin{array}{l}\text { Duration of } \\
\text { PPROM in hrs }\end{array}$ & $\begin{array}{l}\text { No. of cases of } \\
\text { maternal morbidity }\end{array}$ & $\%$ & $\begin{array}{l}P \\
\text { value }\end{array}$ \\
\hline$<24$ & 5 & 9.8 & \multirow[t]{4}{*}{0.001} \\
\hline $24-48$ & 9 & 17.6 & \\
\hline $48-72$ & 16 & 31.3 & \\
\hline$>72$ & 21 & 41.1 & \\
\hline
\end{tabular}

Perinatal mortality was $0.7 \%$ when birth weight was $>2500$ gms. 
Table 5: Analysis of PPROM and perinatal morbidity and perinatal mortality.

\begin{tabular}{|lll|}
\hline Perinatal morbidity: causes & No. of cases & $\%$ \\
\hline Birth asphyxia & 18 & 5.38 \\
\hline RDS & 15 & 4.4 \\
\hline Sepsis & 22 & 6.58 \\
\hline Hyperbilirubinemia & 16 & 4.7 \\
\hline NEC & 4 & 1.19 \\
\hline Total & 75 & 22.4 \\
\hline Perinatal mortality: causes & & \\
\hline Birth asphyxia & 4 & 1.19 \\
\hline RDS & 2 & 0.59 \\
\hline Sepsis & 5 & 1.49 \\
\hline Total & 11 & 3.29 \\
\hline
\end{tabular}

Table 6: Analysis of perinatal morbidity in relation to duration of PPROM.

\begin{tabular}{|c|c|c|c|c|}
\hline $\begin{array}{l}\text { Duration of } \\
\text { PPROM in } \\
\text { hours }\end{array}$ & $\begin{array}{l}\text { No. of } \\
\text { cases }\end{array}$ & $\begin{array}{l}\text { Perinatal } \\
\text { morbidity }\end{array}$ & $\%$ & $\begin{array}{l}\mathrm{P} \\
\text { value }\end{array}$ \\
\hline$<24$ & 61 & 10 & 16.3 & \multirow{4}{*}{0.001} \\
\hline $24-48$ & 122 & 16 & 13.1 & \\
\hline $49-72$ & 65 & 21 & 32.3 & \\
\hline$>72$ & 86 & 28 & 32.5 & \\
\hline $\begin{array}{l}\text { Duration of } \\
\text { PPROM in } \\
\text { hours }\end{array}$ & $\begin{array}{l}\text { No. of } \\
\text { cases }\end{array}$ & $\begin{array}{l}\text { Perinatal } \\
\text { mortality }\end{array}$ & $\%$ & $\begin{array}{l}P \\
\text { value }\end{array}$ \\
\hline$<24$ & 61 & 1 & 1.6 & \multirow{4}{*}{0.47} \\
\hline $24-48$ & 122 & 3 & 2.4 & \\
\hline $49-72$ & 65 & 4 & 6.15 & \\
\hline$>72$ & 86 & 2 & 2.3 & \\
\hline
\end{tabular}

Table 7: Analysis of perinatal morbidity and mortality according to gestational age.

\begin{tabular}{|llllll|}
\hline Weight & No. of cases & Perinatal morbidity & $\%$ & Perinatal mortality & $\%$ \\
\hline$<1000$ & 8 & 6 & 75 & 2 & 25 \\
\hline $1001-1500$ & 44 & 24 & 54.5 & 4 & 9.09 \\
\hline $1501-2000$ & 57 & 19 & 33.3 & 3 & 5.2 \\
\hline $2001-2500$ & 87 & 16 & 18.3 & 1 & 1.1 \\
\hline$>2500$ & 138 & 10 & 7.2 & 1 & 0.7 \\
\hline
\end{tabular}

\section{DISCUSSION}

The present study conducted in a tertiary centre revealed a incidence of PPROM to be $2.2 \%$. This was comparable with the incidence rates of studies conducted by Jayaram et al (3.8\%) and Canavan et al (3\%).,8 But study conducted by Pandey et al revealed an incidence of $7.7 \%$ which was high compared to this study. ${ }^{9}$ The incidence of PPROM at Parkland Memorial Hospital was 1\%. This low rate may be due to better socioeconomic status, regular antenatal checkups, prompt identification and treatment of vaginal infections and other risk factors in developed countries compared to India.

Akter et al found that maximum number of patients with preterm premature rupture of membranes were from 2130 years and mean age was $27.24 \pm 6.28$ years. ${ }^{2}$ Triniti et al also reported the mean age of $29.8 \pm 7.2$ years among patients with preterm premature rupture of membranes. ${ }^{10}$ In this study PPROM was present in $50.1 \%$ of patients in the age group 21-30 years and the mean age was $26.3 \pm 6.9$ years, which was comparable with the above studies.

In this study, the patients of low socio-economic status were $55.2 \%$ and middle socioeconomic status were $29.6 \%$ which was comparable with the study by Swathi Pandey which was $61 \%$ and $39 \%$ respectively. In this study booked cases were $72 \%$ and unbooked cases were
28\%. This was not comparable with the study by Akter et al which shows unbooked cases as $90 \% .^{2}$

Multiparity is a risk factor for PPROM due to long standing infection, previous trauma to cervix and patulous os. In this study $61.1 \%$ were multigravida and $38.9 \%$ were primigravida which was comparable with study by Akter at al (multigravida $62 \%$ primigravida $38 \%) .^{2}$

Highest number of cases $(56.3 \%)$ of PPROM occurred near to term (34-0 to $36-6$ weeks). The mean gestational age at the onset of membrane rupture was $34.1 \pm 2.4$ weeks This was comparable with the study by Akter et al which showed $66 \%$ of cases of PPROM between 35-37 weeks of gestation and mean gestational age of $34.74 \pm 2.27$ weeks. $^{2}$

Merstein et al reported that preterm PROM which occurs before 32 weeks gestation accounts for $20-40 \%$ of PROM and the incidence is double in multiple pregnancy. ${ }^{11}$ In this study $86 \%$ were singleton pregnancies, $12.9 \%$ were twins and $1.06 \%$ were triplets.

In this study, $48.4 \%$ had history of termination of pregnancy, $28.6 \%$ had history of PPROM, $10.5 \%$ had history of LSCS and $4.09 \%$ had history of preterm delivery. This is comparable with a study by Swathi Pandey in which the recurrence of PPROM was $21 \%$ and 
in the data of collaborative perinatal project the risk of recurrence of PPROM in subsequent pregnancy was $21 \%$. The maternal and fetal medicine network found the risk of recurrent PPROM to be $32 \%$. Noor $\mathrm{S}$ et al also concluded that history of previous one or more preterm delivery was significantly associated with PPROM. ${ }^{12}$ The largest effect on subsequent reproduction was produced by multiple prior abortions. The excessive force and greater diameter dilatation may carry increase risk of cervical incompetence and PPROM.

Regarding the mode of delivery, $77.5 \%$ had vaginal delivery, among which $64.5 \%$ had normal vaginal delivery, $2.7 \%$ assisted vaginal delivery (forceps/ventouse), $\quad 10.2 \%$ breech delivery (spontaneous/assisted) and $22.5 \%$ underwent LSCS. This was comparable with the study conducted by Pandey et al who reported the incidence of caesarean section as $31 \%$ in the study group. The mode of delivery according to parity did not show any significant difference according to parity in normal vaginal delivery, but assisted vaginal delivery (ventouse/forceps) was more among primigravida than in multigravida. Vaginal breech delivery and LSCS was found to be more among multigravida than in primigravida. There was no statistical significance in these groups.

Maximum number of LSCS (39.4\%) were seen in the group with bishop's score 0-5. LSCS was significantly more when cervix was unripe compared to cases when bishop's score was more than 5 . Cases of unfavourable cervix can be benefited by local prostaglandin gel which ripen the cervix and induce labour followed by oxytocin supplementation if needed. Also, malpresentations, failure of induction, fetal distress, oligohydramnios resulted in LSCS. In this study $23.6 \%$ of patients underwent LSCS when AFI $<5$ which is comparable with a study conducted by Borna $\mathrm{S}$ et al who reported that patients with $\mathrm{AFI}<5 \mathrm{~cm}$ demonstrated greater frequency of LSCS for non-reassuring fetal tests (23\%vs $2.8 \%){ }^{13}$

As the duration of PPROM inceases, maternal morbidity also increases. The maternal morbidity in this study was $17.4 \%$ which does not correlate with the study by Pandey et al $9 \%$. In the study by Akter et al, maternal morbidity was $1.6 \%$ when the duration of PPROM was $<24$ hours and $12.5 \%$ when the duration of PPROM was $>24$ hours which did not correlate with our study where maternal morbidity was $9.8 \%$ when the duration of PPROM was $<24$ hours and $90 \%$ when the duration of PPROM was $>24$ hours. $^{2}$

In this study, perinatal morbidity is $22.4 \%$ out of which $6.58 \%$ were sepsis, $5.38 \%$ birth asphyxia, $4.4 \%$ RDS. This is comparable with the study by Jayaram et al where perinatal mortality was $24 \% .^{7}$

Regarding the perinatal mortality which was $3.29 \%$ of which $1.49 \%$ were due to sepsis, $1.19 \%$ were due to birth asphyxia and $0.59 \%$ due to RDS. This is not comparable with the study of Pandey et al which showed a perinatal mortality of $12 \% .^{9}$

The high incidence of maternal and neonatal infection may be consequence of decreased antibacterial activity in the amniotic fluid which is low in early pregnancy and increases with gestational age. Another factor is limited ability of preterm infant to fight infection.

In many studies, it was found that the risk of neonatal infection was increased among mothers with premature rupture of membranes $>18$ hours, maternal fever during labour and prematurity. In present study, perinatal morbidity was $16.3 \%$ in PPROM<24 hours and $77.9 \%$ in PPROM $>24$ hours and perinatal mortality was $1.6 \%$ in PPROM $<24$ hours and $10.3 \%$ in PPROM $>24$ hours. The latency from the membrane rupture to delivery interval ranged from $0-72$ days with a mean of $48.4 \pm 6.8$ hours

Perinatal morbidity and mortality increases when the weight at birth decreases. When the weight was $<1000$ gms perinatal mortality was $75 \%$ and mortality was $25 \%$. It was reduced to $7.2 \%$ and $0.7 \%$ when the birth weight was $>2500 \mathrm{gms}$. In this study, neonatal sepsis was seen in 22 cases out of which $63.6 \%$ were $<2000 \mathrm{gms}$, $27.2 \%$ weighed between 2000-2500 gms and 9.2\% were $>2500 \mathrm{gms}$. This was not comparable with the study by collaborative perinatal project in which neonatal sepsis was seen in $20 \%-<2000 \mathrm{gms}, 4.8 \%-2000$ to $2500 \mathrm{gms}$ and $2 \%$ in $>2500 \mathrm{gms}$.

Corticosteroids do play a role in reducing RDS in premature babies. Tocolytics do not have much role in PPROM. These patients could be offered initial labour inhibition to achieve 48 hours of corticosteroids benefit in the absence of infection. Prophylactic antibiotics do not seem to have much role in the prevention of neonatal sepsis. However, they do have some role in the prevention of puerperal sepsis. Perinatal mortality is not influenced by the mode of delivery.

Management selected in PPROM should be one that has least risk to mother and fetus. A gestational age approach to therapy is important and should be adjusted for each hospital's NICU outcome.

\section{CONCLUSION}

PPROM is a significant obstetric problem. Despite exhaustive research most of the aspects of PPROM remain enigmatic. It contributes to increased maternal morbidity as well as perinatal morbidity and mortality. Careful antenatal monitoring, detection and prompt treatment of infection is necessary. Strict aseptic precautions, appropriate therapy, regular follow up are important in the prevention and management of PPROM. Close antenatal monitoring, identification of risk factors like cervicovaginal infection and their management play an important role in the prevention of PPROM. From this 
study, we arrive at the conclusion that management should not be generalised regime. Multifactorial study of individual cases and management has to plan accordingly, varying from expectant to aggressive therapy. Danger of infection to both mother and fetus increases with increased duration of PPROM. Our experience to date from the available resources suggests that management of PPROM still requires critical study.

\section{ACKNOWLEDGMENTS}

The authors are thankful to all the faculty and staff members of the Department of Obstetrics and Gynaecology, RIMS, Imphal, India and the patients for their immense cooperation and support during the study period.

Funding: No funding sources

Conflict of interest: None declared

Ethical approval: The study was approved by the Institutional Ethics Committee

\section{REFERENCES}

1. Brian MM. Preterm Premature Rupture of the Membranes. ACOG. 2003;101:1.

2. Akter S, Akther R, Rashid Z. Preterm prelabour rupture of the membrane and feto-maternal outcome: an observational study. J Bangladesh Coll Phys Surg. 2010;28:1.

3. ACOG Committee on Practice Bulletins-Obstetrics. ACOG Practice Bulletin No. 80: premature rupture of membranes. Clinical management guidelines for obstetrician-gynaecologists. Obstet Gynecol. 2007;109(4):1007-19.

4. Medina TM, Hill DA. Preterm premature rupture of membranes: diagnosis and management. Amer Fam Physician. 2006;73:659-64.

5. Hyagriv NS, Timothy PC. Preterm premature rupture of membranes: diagnosis, evaluation and management strategies. BJOG. 2005;11:32-7.
6. Bottoms SF, Paul RH, Mercer BM, MacPherson CA, Caritis SN, Moawad AH et al. Obstetric determinants of neonatal survival: Antenatal predictors of neonatal survival and morbidity in extremely low birth weight infants. Am J Obstet Gynecol. 1999;180:665-9.

7. Jayaram KV, Sudha S. A study of premature rupture of membranes- Management and outcome. J Obstet Gynecol India. 2001;51:58-60.

8. Canavan TP, Simhan HN, Caritis S. An evidencebased approach to the evaluation and treatment of premature rupture of membranes: Part II. Obstet Gynecol Surv. 2004;59(9):678-89.

9. Pandey S, Dave A, Bandi S. Maternal and fetal outcome in cases of PROM. J Obstet Gynecol India. 2000;50:63-5.

10. Triniti A, Suthatvorawut S, Prasertsawat PO. Epidemiologic study of cervical swab culture in preterm premature rupture of membrane (PPROM) at Ramathibodi Hospital. Thai J Obstet Gynaecol. 2008;16:173-8.

11. Merenstein GB, Weisman LE. Premature rupture of membranes and neonatal consequences. SemeinPerinatol .1996;20:375-80.

12. Noor S, Fawwad A, Shahzad H, Sultana R, Bashir R. Foetomaternal outcome in patients with or without premature rupture of membranes. J Ayub Med Coll Abbottabad. 2010;22:164-7.

13. Borna S, Borna $\mathrm{H}$, Khazardoost $\mathrm{S}$ and Hantoushzadeh S. Perinatal outcome in preterm premature rupture of membranes with amniotic fluid index < 5 (AFI < 5). BMC Pregnancy Childbirth. 2004;4:1471-2393.

Cite this article as: Mohan SS, Thippeveeranna C, Singh NN, Singh LR. Analysis of risk factors, maternal and fetal outcome of spontaneous preterm premature rupture of membranes: a cross sectional study. Int J Reprod Contracept Obstet Gynecol 2017;6:3781-7. 